\title{
BMJ Open Efficacy of pressure ulcer prevention interventions in adult intensive care units: a protocol for a systematic review and network meta-analysis
}

\author{
Yixiu Du, ${ }^{1}$ Fangqin Wu, ${ }^{1}$ Sai Lu, ${ }^{2}$ Wei Zheng, ${ }^{1}$ Huiying Wang, ${ }^{3}$ Ruming Chen, ${ }^{4}$ \\ Xiaoying Lu, ${ }^{4}$ Yu Zhang ${ }^{4}$
}

To cite: Du Y, Wu F, Lu S, et al. Efficacy of pressure ulcer prevention interventions in adult intensive care units: a protocol for a systematic review and network meta-analysis. BMJ Open 2019;9:e026727. doi:10.1136/ bmjopen-2018-026727

- Prepublication history and additional material for this paper are available online. To view these files, please visit the journal online (http://dx.doi org/10.1136/bmjopen-2018026727).

Received 19 September 2018 Revised 22 February 2019 Accepted 28 February 2019

Check for updates

(c) Author(s) (or their employer(s)) 2019. Re-use permitted under CC BY-NC. No commercial re-use. See rights and permissions. Published by BMJ.

${ }^{1}$ School of Nursing, Capital Medical University, Beijing, China

${ }^{2}$ College of Health and Biomedicine, Institute for Health and Sport, Victoria University, Melbourne, Victoria, Australia ${ }^{3}$ Surgical Ward, Hospital of Tsinghua University, Beijing,

China

${ }^{4}$ Heart Center, Beijing Chaoyang Hospital affiliated with Capital

Medical University, Beijing, China

Correspondence to

Fangqin Wu;

fangqin_wu@sina.com

\section{ABSTRACT}

Introduction Pressure ulcers (PUs) are associated with substantial health burden. Patients in intensive care units (ICUs) are at high risk for developing PU. In the absence of large randomised controlled trials (RCTs) that compare commonly known interventions for preventing PU in ICUs, uncertainty remains around the best practice strategy for PU management in adult ICUs. This study, therefore, aims to identify the most effective interventions and combinations of interventions that prevent PU in adult ICU using systematic review and network meta-analysis (NMA).

Methods and analysis We will search for all published and unpublished RCTs evaluating interventions to prevent PU compared with other PU prevention measures or with usual care in adult ICU. The primary outcomes are the incidence of PUs and PU severity in critically ill patients in ICU. The secondary outcomes include number of PUs per patient and intervention-related harms caused by the prevention intervention or intervention-related harms. All data extraction will be performed by at least two independent reviewers on the basis of a priori developed extraction form. We will evaluate the risk of bias of the included RCTs in accordance with the Cochrane Collaboration's risk of bias tool, and assess the quality of evidence using Grading of Recommendations Assessment, Development and Evaluation. A standard pairwise meta-analysis and a Bayesian NMA will be conducted to compare the efficacy of different PU prevention interventions. A surface under the cumulative ranking curve will be used to rank the probabilities of each prevention intervention for various outcomes.

Ethics and dissemination This study will not require the ethics approval as it is a review based on published studies. The findings of this study will be submitted to a peer-reviewed journal for publication. We anticipate that the results of the study will provide the evidence to inform clinicians and guideline developers on determining the best interventions for the prevention of PU in ICU patients. PROSPERO registration number CRD42018085562.

\section{INTRODUCTION}

Hospital acquired pressure ulcers (HAPUs) are a significant global challenge associated with considerable physical and economic
Strengths and limitations of this study

- To the best of our knowledge, this study will be the first network meta-analysis that comprehensively explores and compares the effectiveness of various pressure ulcer prevention interventions in adult intensive care unit (ICU) patients.

- The present study will ensure that both the risk of bias and the quality of evidence of the included randomised controlled trial is properly assessed by Cochrane risk of bias assessment tool and Grading of Recommendations Assessment, Development and Evaluation, respectively.

- The findings from this study may help guide healthcare providers to select appropriate prevention methods which may ultimately lead to a reduction in healthcare costs and improved patient outcomes in adult ICUs.

- Due to the retrospective nature of this study, the findings of this study are likely to be influenced by the quantity and quality of the studies included.

burden. ${ }^{1-3}$ Critically ill patients in intensive care units (ICU) are at high risk of developing HAPU due to their characteristics such as multiple comorbidities, unstable haemodynamics, bedridden, increased use of medical devices and special medications. ${ }^{4-8}$ Estimates suggest that up to $49 \%$ of the ICU patients developed HAPUs. ${ }^{9-14}$

Previous research including systematic reviews and meta-analyses have failed to reach robust conclusions on the relative effectiveness of different PU prevention strategies due to the limitation of not allowing for indirect comparisons. ${ }^{6}{ }^{15-20}$ For instance, a recent systemic review of 25 studies on the effectiveness of PU prevention strategies in the ICU cautiously recommended a silicone foam dressing, however, no single superior strategy was identified in the intensive care context. ${ }^{20}$ McInnes and colleagues conducted a meta-analysis with aims to assess the effects 
of supporting surfaces in preventing PUs. The authors selected 53 trials with more than 16000 participants. They found several known surface supporting devices appear to have no clear benefits in practice ${ }^{21}$ Consequently, a variance continues to exist between recommended PU preventions and actual practice. ${ }^{22}$

Network meta-analysis (NMA) is a statistical methodology that compares multiple interventions simultaneously by analysing studies making different comparisons in the same analysis. ${ }^{23}$ Contrary to the traditional meta-analysis, NMA incorporates direct and indirect evidence within a network of RCTs and enables comparison of multiple intervention and ranking of the interventions that have not been studies in a head to head fashion. ${ }^{24}$ Despite that PU prevention programmes encompass a variety of measures such as education, training, repositioning, nutritional support, moisture management and support surfaces, ${ }^{15}$ 17-19 25-27 only the effectiveness of support surfaces was recently assessed by an NMA. ${ }^{28} \mathrm{Up}$-to-date, no NMA has been conducted to systematically compare and rank the efficacy of the available prevention strategies in adult ICU patients, this study is therefore deemed a necessity. The objective of the study is to rank the effectiveness of PU prevention interventions in ICUs through a systematic review and NMA. The review question is, what are the most effective PU prevention interventions (or combinations of interventions) compared with usual care for reducing the incidence of PU in adult ICUs?

\section{METHODS AND ANALYSIS}

Eligibility criteria

Types of settings

Only studies conducted in adult ICUs will be included.

\section{Types of participants}

Patients aged 18 years or older in adult ICUs will be included. Patients will not be eligible if they have been diagnosed with PU prior to their admission to the ICU. No further restrictions will be made on participants' gender, ethnicity and nationality.

Types of studies

Only full articles of RCTs reporting comparisons of one prevention intervention with another or conventional care for adult ICU patients will be included. We will exclude editorials, opinion papers, reviews, qualitative studies and case reports. Quasiexperimental and non-experimental such as cohort, case-control and pre-post studies will also be excluded.

\section{Types of intervention}

We are interested in any intervention aimed at preventing PU in adult ICU patients. Both monofactorial and multifactorial interventions will be included, such as risk assessment, support surfaces, dressings, repositioning regiments, nutritional supplementation and educational or training programmes.
Comparators

We will make comparisons between the usual care and single prevention measure as well as between different PU prevention interventions.

\section{Outcome measures}

\section{Primary outcomes}

1. The incidence of PUs in critically ill patients in ICUs. This will be calculated based on the percentage of the participants who develop new PUs during the study period. ${ }^{29}$

2. The severity of PUs. This will be classified as per the National Pressure Ulcer Advisory Panel (NPUAP), European Pressure Ulcer Advisory Panel (EPUAP), and Pan Pacific Pressure Injury Alliance (PPPIA) Clinical Practice Guidelines. ${ }^{30}$

\section{Secondary outcomes}

1. Number of PUs per patient.

2. Adverse events caused by the prevention intervention or intervention-related harms.

The literature search will be limited to English and Chinese articles and no date restrictions will be applied. We are aware that overlooking non-English and non-Chinese articles may lead to biases and gaps in our understanding of the topic. However, the literature has shown the dominance of English in international scientific publications with almost $80 \%$ research findings reported in English. ${ }^{31}$ Considering the dramatic increase in scientific publications in Chinese-language journals, ${ }^{32}$ encompassing both English and Chinese articles may allow us to achieve a descent coverage. A draft eligibility form (online appendix 1) has been developed based on the relevant study. ${ }^{33}$ This form will be further reviewed by a panel of experts comprising librarian, ICU clinical nurse specialists, wound care nurse specialists and subsequently piloted by the key investigators.

\section{Search strategy}

'Pressure ulcer', 'pressure sore', 'pressure injury', 'decubitus', 'decubitus ulcers', 'bed sore', 'intensive care' and 'critical care' will be used as keywords or (and) MeSH terms. Searches will be undertaken in the following electronic databases: EMBASE, PubMed, the Cochrane Central Register of Controlled Trials, CINAHL, SinoMED to identify published studies. The databases will be searched from their inception to 31 January 2019. An experienced librarian will be invited to review the selected search strategy. A draft search strategy is summarised in online appendix 2. We will also contact organisations that produce clinical practice guideline of PU management (eg, NPUAP, npuap.org; EPUAP, epuap.org; PPPIA; Chinese Nursing Association; Japanese Society for Pressure Ulcers). In addition, we will manually search for the unpublished literature and check references of all included trials to ensure relevant articles are covered. Experts in the PU prevention and management field 


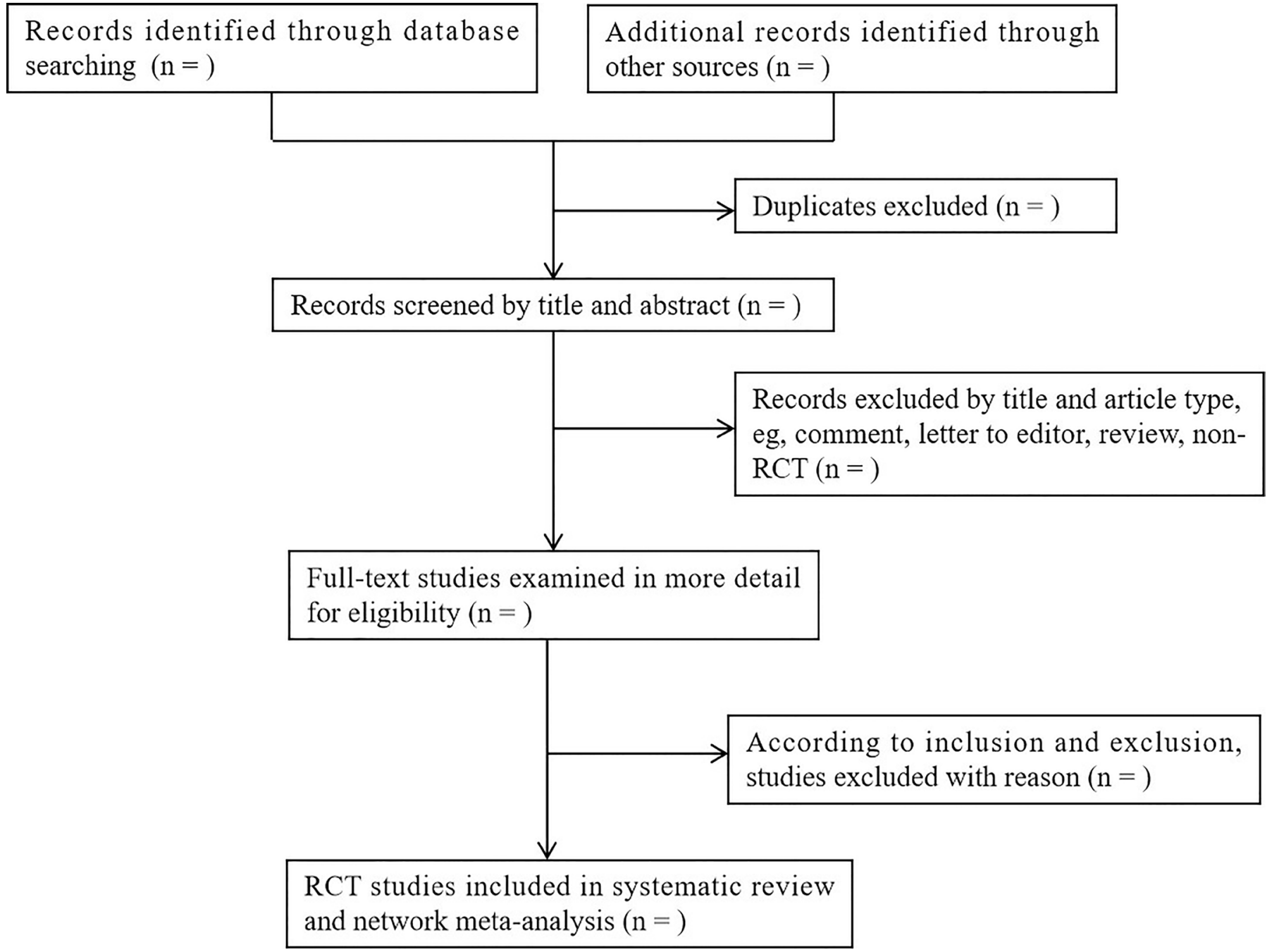

Figure 1 Study selection process. RCT, randomised controlled trial.

will also be contacted via email to locate non-published studies.

\section{Study selection}

All investigators will be properly trained prior to the commencement of the data screening. Rayyan online literature management software (https://rayyan.qcri.org) will also be introduced to screen and manage literature. After screening a random sample of 50 citations, Kappa test will be used to calculate the inter-observer agreement for all studies included. If Kappa value is less than 0.75, a second round of training will be implemented. Duplicate publications of original research will be excluded. The titles and abstracts of identifiable articles will be screened independently by two reviewers (YD and FW), to exclude reports that clearly do not meet the inclusion criteria. The same reviewers will then independently examine fulltext articles to determine eligibility. When disagreements arise, the third author (WZ) will be asked to evaluate the full text and the discrepancy will be resolved by group discussions. Excluded trials and the rationale for exclusion will be recorded. Figure 1 depicts the study selection processes in a Preferred Reporting Items for Systematic Reviews and Meta-Analyses (PRISMA) ${ }^{34}$ flow diagram.

\section{Data extraction}

A rigorous process will be applied to the data extraction. First, a draft data extraction form will be developed through team discussions. The form will then be pilot tested by each member of the team prior to its usage. Two reviewers (YD and FW) will extract the data of interest independently using the data extraction form, and conflicts or disagreements will be resolved by team discussions. The following descriptive data will be extracted: country of origin, author(s), year of publication, intervention(s) and comparator(s), sample size, patient characteristics, setting, primary outcomes, secondary outcomes, outcome measurements and any adverse events caused by the preventive interventions. In case of insufficient information, wherever possible, authors of primary studies will be contacted by either telephone, email or post to obtain missing data. If a study presents incomplete primary outcomes it will only be included in the systematic review rather than the meta-analysis. 


\section{Quality appraisal}

Risk of bias appraisal

Cochrane Risk of Bias Assessment Tool will be used to appraise the risk of bias. ${ }^{35}$ This tool includes: (1) random sequence generation (selection bias); (2) allocation concealment (selection bias); (3) blinding of outcome assessors (detection bias); (4) blinding of patients and personnel (performance bias); (5) incomplete outcome data (attrition bias); (6) selective reporting (reporting bias); (7) anything else, ideally prespecified (other bias). Authors of the original study will be contacted for more information if there are inadequate details of allocation concealment and other characteristics of trials. Each item will be classified into high risk or unclear risk or low risk with respect to the level of risk of bias by two independent reviewers (YD and FW). Any discrepancies will be resolved by a third reviewer.

\section{Geometry of the network}

The available evidence will be presented in a network plot to ensure it provides information on the relative effectiveness of PU prevention measures. The size of the nodes will reflect the sample size of each intervention and the thickness of edges will be associated with numbers of RCTs.

\section{Data synthesis and statistical analysis \\ Pairwise meta-analysis}

Data will be synthesised with a pairwise meta-analysis. Risk ratio (RR) and 95\% CI will be used for dichotomous outcomes, mean differences or standardised mean differences (SMDs) with $95 \%$ CI will be used for continuous outcomes. We will evaluate clinical and methodological heterogeneity through examination of the population, methods and interventions of the included studies. Heterogeneity across trials will be assessed by estimating the variance between studies using the $\chi^{2}$ test and $\mathrm{I}^{2}$ statistic. For a $\mathrm{p}$ value $\geq 0.1$ and $\mathrm{I}^{2} \leq 50 \%$, no significant statistical heterogeneity exists and the MantelHaenszel fixed effect model will be employed; otherwise the Mantel-Haenszel random effects model will be used ( $\mathrm{p}$ value $<0.1$ and $\mathrm{I}^{2}>50 \%$ ). The sources of heterogeneity will be explored by sensitivity analyses. ${ }^{36}$ The potential for publication bias will be examined through the Begg's and Egger's test if more than 10 studies are available. The contour-enhanced funnel plot will be obtained additionally to distinguish asymmetry. The pairwise meta-analysis will be carried out in Stata V.13.

\section{Evaluation of the transitivity assumption}

Transitivity is one of the major assumptions that NMA relies upon. ${ }^{24}$ We will assess the transitivity by checking whether time to occurrence of PUs is similar in the different RCTs and comparing the distribution of the potential modifiers such as age, and the risk scale of PU across the different pairwise comparisons. ${ }^{23}$

\section{Network meta-analysis}

We will perform Bayesian NMAs to compare the efficacy of selected PU prevention interventions. The NMA will be conducted in the WinBUGS V.1.43 software (Medical Research Council Biostatistics Unit, Cambridge, UK). ${ }^{37}$ A complex statistical models will be built by a Bayesian software program, the Markov Chains Monte Carlo simulation technique will be used to generate samples. An initial burn-in period of 5000 iterations will be set to allow convergence, then posterior summaries will be produced based on a further 30000 iterations. Both Kernel density and the auto-correlation plots will be used to assess model convergence. ${ }^{38}$ We will calculate the summary RRs or SMD for all pairwise comparisons in a league table. The ranking probabilities of all prevention interventions for each outcome will be estimated via the surface under the cumulative ranking curve. ${ }^{39}$ We will also compare stability using the frequentist approach. ${ }^{40}$

\section{Assessment of inconsistency}

The consistency between direct and indirect evidence will be assessed by a loop-specific approach within each loop of the network. The loop-specific approach separates direct evidence from indirect evidence for a specific comparison in the loop (inconsistency factor). $\mathrm{I}^{2}$ and its $95 \%$ CI will be used to estimate the consistency within the entire network. ${ }^{41}$ If the inconsistency is identified, subgroup analyses and multiple meta-regressions will be performed to determine the impact of the mean age of the participants, the risk score of PU, the length of ICU stay and blinding method on the PU incidence.

\section{Rating the confidence in estimates of the effect in NMA}

The quality of evidence that influences network estimates will be assessed using the Grading of Recommendations Assessment, Development and Evaluation. ${ }^{42}$ On the basis of five domains including the study limitations, inconsistency, imprecision, indirectness and publication bias, the quality of evidence will be classified into one of the four levels, including high, moderate, low and very low quality. ${ }^{42}$

\section{Patient and public involvement}

Patients and the public were not involved in the conception and design of this protocol.

\section{Ethics and dissemination}

Ethical approval is not required for this study as it does not include any interventions with human or animal or confidential personal data. The findings of this study may assist clinicians and guideline developers in their efforts to prevent PU in ICU patients. The procedures of the study will be conducted in accordance with the PRISMA-compliant guidelines. The findings of the study will be reported according to the PRISMA-compliant guidelines and submitted to a peer-reviewed journal for publication.

Acknowledgements We would like to thank Associate Professor Karen V. Lamb, College of Nursing, Rush University, for her invaluable assistance in the language editing. 
Contributors After conceptualising and designing of the study, FW and YD registered the protocol on the PROSPERO database. YD, FW and SL critically revised the protocol and contributed to the drafting of the final manuscript. YD, WZ, HW, $\mathrm{RC}, \mathrm{XL}$ and $\mathrm{YZ}$ tested the feasibility of the study and involved in the revision of the protocol. YD, FW, WZ, HW and YZ will perform the data collection and analyses. All authors read and approved the final manuscript.

Funding The authors have not declared a specific grant for this research from any funding agency in the public, commercial or not-for-profit sectors.

Competing interests None declared.

Patient consent for publication Not required

Provenance and peer review Not commissioned; externally peer reviewed.

Open access This is an open access article distributed in accordance with the Creative Commons Attribution Non Commercial (CC BY-NC 4.0) license, which permits others to distribute, remix, adapt, build upon this work non-commercially, and license their derivative works on different terms, provided the original work is properly cited, appropriate credit is given, any changes made indicated, and the use is non-commercial. See: http://creativecommons.org/licenses/by-nc/4.0/.

\section{REFERENCES}

1. Lourenco L, Blanes L, Salomé GM, et al. Quality of life and selfesteem in patients with paraplegia and pressure ulcers: a controlled cross-sectional study. J Wound Care 2014;23:331-7.

2. Becker D, Tozo TC, Batista SS, et al. Pressure ulcers in ICU patients: Incidence and clinical and epidemiological features: A multicenter study in southern Brazil. Intensive Crit Care Nurs 2017;42:55-61.

3. Bo M, Massaia M, Raspo S, et al. Predictive factors of in-hospital mortality in older patients admitted to a medical intensive care unit. $J$ Am Geriatr Soc 2003;51:529-33.

4. Edsberg LE, Langemo D, Baharestani MM, et al. Unavoidable pressure injury: state of the science and consensus outcomes. $J$ Wound Ostomy Continence Nurs 2014;41:313-34.

5. Bly D, Schallom M, Sona C, et al. A model of pressure, oxygenation, and perfusion risk factors for pressure ulcers in the Intensive Care Unit. Am J Crit Care 2016;25:156-64.

6. Keller BP, Wille J, van Ramshorst B, et al. Pressure ulcers in intensive care patients: a review of risks and prevention. Intensive Care Med 2002;28:1379-88.

7. Kaewprag P, Newton C, Vermillion B, et al. Predictive models for pressure ulcers from intensive care unit electronic health records using Bayesian networks. BMC Med Inform Decis Mak 2017;17(Suppl 2):65

8. Lima Serrano M, González Méndez MI, Carrasco Cebollero FM, et al Risk factors for pressure ulcer development in Intensive Care Units: A systematic review. Med Intensiva 2017;41:339-46.

9. Coyer F, Miles S, Gosley S, et al. Pressure injury prevalence in intensive care versus non-intensive care patients: a state-wide comparison. Aust Crit Care 2017;30:244-50.

10. Jiang $Q$, Li X, Qu X, et al. The incidence, risk factors and characteristics of pressure ulcers in hospitalized patients in China. Int $J$ Clin Exp Pathol 2014;7:2587-94.

11. Lachenbruch $\mathrm{C}$, Ribble $\mathrm{D}$, Emmons $\mathrm{K}$, et al. Pressure ulcer risk in the incontinent patient: analysis of incontinence and hospital-acquired pressure ulcers from the International Pressure Ulcer Prevalence ${ }^{\mathrm{TM}}$ Survey. J Wound Ostomy Continence Nurs 2016;43:235-41.

12. Gunningberg L, Donaldson N, Aydin C, et al. Exploring variation in pressure ulcer prevalence in Sweden and the USA: benchmarking in action. J Eval Clin Pract 2012;18:904-10.

13. Bauer K, Rock K, Nazzal M, et al. Pressure Ulcers in the United States' Inpatient Population from 2008 to 2012: results of a retrospective Nationwide Study. Ostomy Wound Manage 2016;62:30-8.

14. El-Marsi J, Zein-El-Dine S, Zein B, et al. Predictors of pressure injuries in a Critical Care Unit in Lebanon: prevalence, characteristics, and associated factors. J Wound Ostomy Continence Nurs 2018;45:131-6.

15. Hommel A, Gunningberg L, Idvall E, et al. Successful factors to prevent pressure ulcers - an interview study. J Clin Nurs 2017;26(12):182-9.

16. Tayyib N, Coyer F, Lewis P. Pressure ulcers in the adult intensive care unit: a literature review of patient risk factors and risk assessment scales. J Nurs Educ Pract 2013;3:28-42.

17. Santamaria N, Gerdtz M, Liu W, et al. Clinical effectiveness of a silicone foam dressing for the prevention of heel pressure ulcers in critically ill patients: Border II Trial. J Wound Care 2015;24:340-5.
18. Walker R, Huxley L, Juttner M, et al. A pilot randomized controlled trial using prophylactic dressings to minimize sacral pressure injuries in high-risk hospitalized patients. Clin Nurs Res 2017;26:484-503.

19. Mclnnes E, Jammali-Blasi A, Bell-Syer SE, et al. Support surfaces for pressure ulcer prevention. Cochrane Database Syst Rev 2015;3:CD001735.

20. Tayyib N, Coyer F. Effectiveness of pressure ulcer prevention strategies for adult patients in intensive care units: a systematic review. Worldviews Evid Based Nurs 2016;13:432-44.

21. Mclnnes E, Jammali-Blasi A, Bell-Syer S, et al. Preventing pressure ulcers--Are pressure-redistributing support surfaces effective? A Cochrane systematic review and meta-analysis. Int J Nurs Stud 2012;49:345-59.

22. Lawrence P, Fulbrook P, Miles S. A survey of Australian nurses' knowledge of pressure injury/pressure ulcer management. JWOCN 2015;42:450-60.

23. Petticrew M, Rehfuess E, Noyes J, et al. Synthesizing evidence on complex interventions: how meta-analytical, qualitative, and mixed-method approaches can contribute. J Clin Epidemiol 2013;66:1230-43.

24. Salanti G. Indirect and mixed-treatment comparison, network, or multiple-treatments meta-analysis: many names, many benefits, many concerns for the next generation evidence synthesis tool. Res Synth Methods 2012;3:80-97.

25. Atkinson RA, Cullum NA. Interventions for pressure ulcers: a summary of evidence for prevention and treatment. Spinal Cord 2018;56:186-98.

26. Haesler E, Kottner J, Cuddigan J. 2014 International Guideline Development Group. The 2014 International Pressure Ulcer Guideline: methods and development. J Adv Nurs 2017;73:1515-30.

27. Behrendt R, Ghaznavi AM, Mahan M, et al. Continuous bedside pressure mapping and rates of hospital-associated pressure ulcers in a medical intensive care unit. Am J Crit Care 2014;23:127-33.

28. Shi C, Dumville JC, Cullum N. Support surfaces for pressure ulcer prevention: a network meta-analysis. PLoS One 2018;13:e0192707.

29. Baharestani MM, Black JM, Carville K, et al. Dilemmas in measuring and using pressure ulcer prevalence and incidence: an international consensus. Int Wound J 2009;6:97-104.

30. Haesler E, ed. National Pressure Ulcer Advisory Panel, European Pressure Ulcer Advisory Panel and Pan Pacific Pressure Injury Alliance. Prevention and treatment of pressure ulcers: quick reference guide. Osborne Park, Australia: Cambridge Media, 2014.

31. Hamel ER. The dominance of English in the international scientific periodical literature and the future of language use in science. AILA Review 2008;20:53-71.

32. Xie Q, Freeman RB. Bigger Than You Thought: China's Contribution to Scientific Publications. https://ideas.repec.org/p/nbr/nberwo/ 24829.html (Accessed 27 Nov 2018).

33. Tricco AC, Cogo E, Holroyd-Leduc J, et al. Efficacy of falls prevention interventions: protocol for a systematic review and network metaanalysis. Syst Rev 2013;2:38.

34. Moher D, Liberati A, Tetzlaff J, et al. Preferred reporting items for systematic reviews and meta-analyses: the PRISMA statement. $J$ Clin Epidemiol 2009;62:1006-12.

35. Higgins JP, Altman DG, Gøtzsche PC, et al. The Cochrane collaboration's tool for assessing risk of bias in randomised trials. BMJ 2011;343:d5928.

36. Dwamena BA. MIDAS: Statamodule for meta-analytical integration of diagnostic accuracy studies. http:// econpapers. repec. org/software/ bocbocode/ s456880. htm (Accessed 8 Jan 2018).

37. Lunn DJ, Thomas A, Best N, et al. Winbugs- a bayesian modelling framework: concepts, structure, and extensibility. Stat Comput 2000;10:325-37.

38. Gelman A, Rubin DB. Inference from Iterative simulation using multiple sequences. Statistical Science 1992;7:457-72.

39. Salanti G, Ades AE, loannidis JP. Graphical methods and numerical summaries for presenting results from multipletreatment meta-analysis: an overview and tutorial. J Clin Epidemiol 2011;64:163-71.

40. Hoaglin DC, Hawkins N, Jansen JP, et al. Conducting indirecttreatment-comparison and network-meta-analysis studies: report of the ISPOR Task force on indirect treatment comparisons good research practices: part 2. Value Health 2011;14:429-37.

41. Jackson D, Barrett JK, Rice S, et al. A design-by-treatment interaction model for network meta-analysis with random inconsistency effects. Stat Med 2014;33:3639-54.

42. Puhan MA, Schünemann HJ, Murad MH, et al. A GRADE Working Group approach for rating the quality of treatment effect estimates from network meta-analysis. BMJ 2014;349:g5630. 